\title{
General base-general acid catalysis by terpenoid cyclases
}

\author{
Travis A Pemberton ${ }^{1}$ and David W Christianson ${ }^{1,2}$
}

Terpenoid cyclases catalyze the most complex reactions in biology, in that more than half of the substrate carbon atoms often undergo changes in bonding during the course of a multistep cyclization cascade that proceeds through multiple carbocation intermediates. Many cyclization mechanisms require stereospecific deprotonation and reprotonation steps, and most cyclization cascades are terminated by deprotonation to yield an olefin product. The first bacterial terpenoid cyclase to yield a crystal structure was pentalenene synthase from Streptomyces exfoliatus UC5319. This cyclase generates the hydrocarbon precursor of the pentalenolactone family of antibiotics. The structures of pentalenene synthase and other terpenoid cyclases reveal predominantly nonpolar active sites typically lacking amino acid side chains capable of serving general base-general acid functions. What chemical species, then, enables the Brønsted acid-base chemistry required in the catalytic mechanisms of these enzymes? The most likely candidate for such general base-general acid chemistry is the co-product inorganic pyrophosphate. Here, we briefly review biological and nonbiological systems in which phosphate and its derivatives serve general base and general acid functions in catalysis. These examples highlight the fact that the Brønsted acid-base activities of phosphate derivatives are comparable to the Brønsted acid-base activities of amino acid side chains.

The Journal of Antibiotics (2016) 69, 486-493; doi:10.1038/ja.2016.39; published online 13 April 2016

\section{INTRODUCTION}

The greater family of terpenoids, including steroids and carotenoids, currently includes more than 75000 members, and new terpenoids continue to be discovered to this day. The vast chemodiversity of this family of natural products arises in large part from the catalytic versatility of terpenoid cyclases. These enzymes catalyze complex cyclization cascades, typically proceeding through multiple carbocation intermediates, to generate myriad hydrocarbon skeletons containing multiple rings and stereocenters. ${ }^{1-5}$ Terpenoid cyclases generally belong to one of two classes, depending on the chemistry that initiates the cyclization cascade: a class I terpenoid cyclase triggers the metaldependent ionization of an isoprenoid diphosphate, such as the sesquiterpene $\left(C_{15}\right)$ farnesyl diphosphate $(\mathrm{FPP})$, to generate an allylic carbocation plus inorganic pyrophosphate $\left(\mathrm{PP}_{\mathrm{i}}\right)$; a class II terpenoid cyclase generates an initial tertiary carbocation by protonation of a carbon-carbon double bond. ${ }^{6-10}$ This Review focuses exclusively on class I terpenoid cyclases, specifically with regard to general basegeneral acid catalysis that facilitates proton transfer steps subsequent to initial carbocation formation.

Pentalenene synthase from Streptomyces exfoliatus UC5319 is a prototypical class I terpenoid cyclase that catalyzes the cyclization of FPP to form pentalenene, which comprises the first committed step in the biosynthesis of the pentalenolactone family of antibiotics (Figure 1). ${ }^{11-13}$ The biological activity of pentalenolactone against Gram-positive and Gram-negative bacteria was discovered nearly 60 years ago, ${ }^{14}$ and the molecular basis for this activity is the inhibition of the glycolytic enzyme glyceraldehyde-3-phosphate dehydrogenase. ${ }^{15,16}$ The stereospecific formation of the tricyclic pentalenene precursor is critical for the antibacterial activity of pentalenolactone, and the three fused rings and four stereocenters of pentalenene are precisely generated in a single reaction catalyzed by pentalenene synthase. Notably, this is the enzyme that first brought the Cane and Christianson laboratories together for a decades-long collaboration on terpenoid cyclase structure and function.

Cane et al. ${ }^{12,13,17}$ reported the preparation of cell-free Streptomyces extracts of pentalenene synthase, enabling enzymological studies with isotopically labeled substrates to elucidate the cyclization mechanism. Notable features of the mechanism first proposed for pentalenene synthase include ionization of FPP, cyclization and stereospecific deprotonation to form the intermediate humulene; this proton is subsequently utilized to reprotonate the adjacent carbon atom to generate the bicyclic protoilludyl cation, which is proposed to undergo hydride transfer, carbon-carbon bond formation and a final stereospecific proton abstraction to yield pentalenene. As noted by

${ }^{1}$ Roy and Diana Vagelos Laboratories, Department of Chemistry, University of Pennsylvania, Philadelphia, PA, USA and ${ }^{2}$ Radcliffe Institute for Advanced Study, and Department of Chemistry and Chemical Biology, Harvard University, Cambridge, MA, USA

Dedicated to Professor David E Cane, with profound gratitude for sharing his wisdom and friendship in more than two decades of collaboration on the structural biology and chemistry of terpenoid biosynthesis.

Correspondence: Professor DW Christianson, Roy and Diana Vagelos Laboratories, Department of Chemistry, University of Pennsylvania, 231 South 34th Street, Philadelphia, PA 19104-6323, USA.

E-mail: chris@sas.upenn.edu

Received 18 January 2016; revised 22 February 2016; accepted 2 March 2016; published online 13 April 2016 


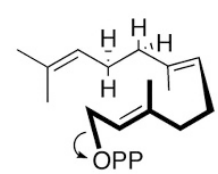

farnesyl diphosphate

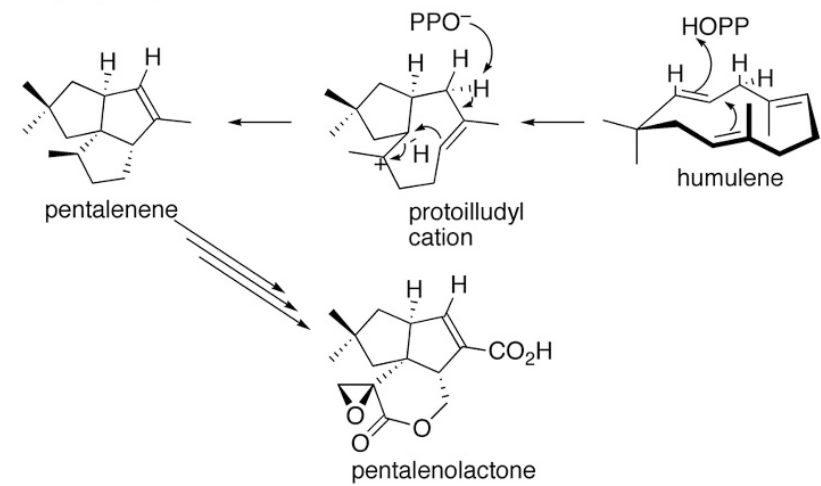

Figure 1 Farnesyl diphosphate cyclization reaction catalyzed by pentalenene synthase from Streptomyces exfoliatus UC5319, as initially proposed by Cane et al. ${ }^{18}$ This reaction is the first step in the biosynthesis of the antibiotic pentalenolactone. The co-product inorganic pyrophosphate ( $\mathrm{PPO}^{-}$, that is, the $\mathrm{PP}_{\mathrm{i}}$ anion) is proposed to be the general base-general acid that mediates stereospecific proton transfers in catalysis.

Cane et al., ${ }^{18}$ a single general base-general acid in the active site would be geometrically competent to mediate the stereospecific deprotonation-reprotonation-deprotonation sequence in the multistep cyclization cascade (Figure 1).

The cloning and expression of pentalenene synthase from S. exfoliatus UC5319 yielded an enzyme identical to the native enzyme in all respects ${ }^{18}$ and ultimately led to the first crystal structure determination of a bacterial terpenoid cyclase. ${ }^{19}$ The enzyme active site was found to be predominantly nonpolar, consistent with the accommodation of the lipophilic substrate. Even so, a single polar residue was observed near the mouth of the active site, $\mathrm{H} 309$, and this residue was hypothesized to be the stereospecific general base-general acid required for pentalenene formation. ${ }^{19}$ However, Cane et al. demonstrated that H309 mutants of pentalenene synthase exhibit nearly full activity. ${ }^{20}$ Mutagenesis of other, less conventional general base candidates, for example, W308 (a tryptophan had been suggested as a catalytic general base in another sesquiterpene cyclase, 5-epiaristolochene synthase $\mathrm{e}^{21}$ ), also proved fruitless. ${ }^{22}$ Where, then, is the general base-general acid that mediates stereospecific proton transfer and the final proton abstraction in the cyclization mechanism?

This question has persisted in the analysis of structure-function relationships ever since the first crystal structures of class I terpenoid cyclases were reported. ${ }^{19,21}$ The first crystal structure determination of a fungal terpenoid cyclase, aristolochene synthase from Penicillium roqueforti, revealed a similar $\alpha$-helical fold to that of pentalenene synthase, and a similarly barren, nonpolar active site devoid of an obvious general base-general acid. ${ }^{23}$ While an active site tyrosine was considered as a possible general base-general acid, mutagenesis studies with the related aristolochene synthase from Aspergillus terreus demonstrated that the corresponding tyrosine was not required for catalytic activity. ${ }^{24}$ Another possible candidate suggested for the general base in $P$. roqueforti aristolochene synthase was the diphosphate leaving group, ${ }^{23}$ and structural studies of A. terreus aristolochene synthase provided compelling structural data that implicated the product $\mathrm{PP}_{\mathrm{i}}$ anion as a general base-general acid. ${ }^{25}$

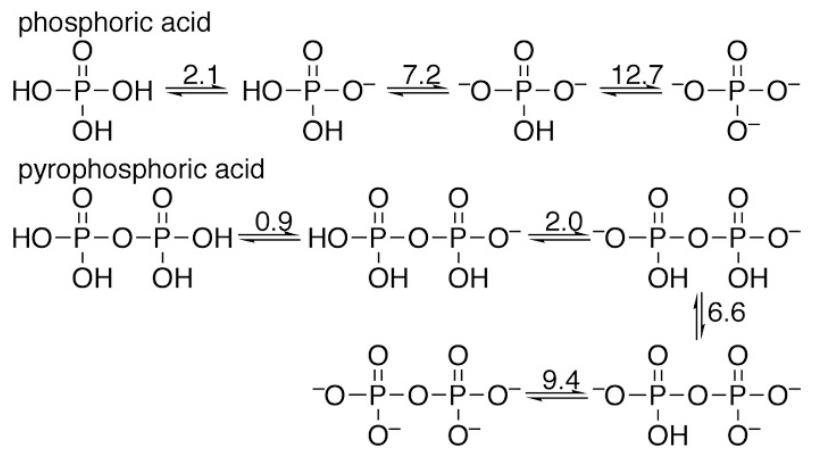

Figure 2 Ionization states and $p K a$ values of phosphoric acid (inorganic phosphate) and pyrophosphoric acid (inorganic pyrophosphate).

Subsequent structural and enzymological studies of aristolochene synthase and other terpenoid synthases likewise implicated the $\mathrm{PP}_{\mathrm{i}}$ anion in general base-general acid catalysis. ${ }^{26-29}$

Given that the $\mathrm{PP}_{\mathrm{i}}$ anion is a co-product for all class I terpenoid cyclase reactions, the possibility of its regular participation in catalysis is intriguing to consider. Following FPP ionization, the $\mathrm{PP}_{\mathrm{i}}$ anion is locked in place by $3 \mathrm{Mg}^{2+}$ ions and 3-4 hydrogen bond interactions, ${ }^{30}$ as first revealed in the crystal structure of the trichodiene synthase$\mathrm{Mg}^{2+}{ }_{3}-\mathrm{PP}_{\mathrm{i}}$ complex. ${ }^{31}$ Ordinarily, inorganic phosphate $\left(\mathrm{P}_{\mathrm{i}}\right)$ and $\mathrm{PP}_{\mathrm{i}}$ might not be considered as routine participants in general base catalysis, even though the range of $\mathrm{pKa}$ values characterizing their various ionization states are comparable to those of amino acid side chains (Figure 2). Moreover, these pKa values can be further modulated by the protein environment, for example, by hydrogen bond interactions and/or metal ion coordination interactions. However, since a tertiary carbocation is quite acidic, with a $\mathrm{pKa}$ of approximately -10 or even lower, a general base in a terpenoid cyclase active site is perhaps not needed so much for activating a sluggish deprotonation step, but instead simply for accepting a highly reactive proton that has nowhere else to go.

Also influencing the stereospecificity of deprotonation, of course, is the conformation of the carbocation intermediate, since the empty $2 p$ orbital of the carbocation must be aligned with the breaking $\mathrm{C}-\mathrm{H}$ bond to facilitate proton loss and $\pi$ bond formation. The threedimensional contour of the terpenoid cyclase active site thus additionally influences carbocation acidity and the stereospecificity of proton transfer steps by controlling the conformations of macrocyclic intermediates.

The first direct structural clue regarding the feasibility of the $\mathrm{PP}_{\mathrm{i}}$ co-product serving as a general base in terpenoid biosynthesis was provided by the X-ray crystal structure of FPP synthase from Escherichia coli complexed with $3 \mathrm{Mg}^{2+}$ ions, isopentenyl diphosphate (IPP), and the nonreactive substrate analog dimethylallyl-Sthiolodiphosphate. ${ }^{32}$ This enzyme is not a terpenoid cyclase, but instead an isoprenoid chain elongation enzyme: it catalyzes successive coupling reactions of dimethylallyl diphosphate with two molecules of IPP to generate FPP. Even so, the structure of this enzyme is homologous to that of class I terpenoid cyclases. ${ }^{33}$ The structure of the FPP synthase- $\mathrm{Mg}^{2+}{ }_{3}$-dimethylallyl-S-thiolodiphosphate-IPP complex revealed that the free (that is, not complexed to $\mathrm{Mg}^{2+}$ ) oxygen atom of the dimethylallyl-S-thiolodiphosphate thiolodiphosphate group is oriented toward the pro-R hydrogen on C2 of IPP, as if poised for the stereospecific proton abstraction that terminates the chain elongation reaction (Figure 3). More recently, the binding of farnesyl-S-thiolodiphosphate as well as aza analogs of carbocation intermediates to aristolochene synthase from Aspergillus terreus 
revealed that a free oxygen of the $\mathrm{PP}_{\mathrm{i}}$ anion is ideally positioned for regiospecific and stereospecific proton abstraction from carbocation intermediates, ${ }^{34}$ consistent with the results of earlier structural studies. $^{25}$ These results strongly suggest that the substrate itself provides useful chemical functionality - the diphosphate/ $\mathrm{PP}_{\mathrm{i}}$ anionfor general base-general acid chemistry in the active site of a class I terpenoid cyclase.

\section{PP I AND P IN GENERAL ACID-GENERAL BASE CATALYSIS}

While phosphate or diphosphate anions such as $\mathrm{P}_{\mathrm{i}}$ or $\mathrm{PP}_{\mathrm{i}}$ are not often considered for general base or general acid functions in enzyme active



Figure 3 The active site of $E$. coli farnesyl diphosphate synthase complexed with three $\mathrm{Mg}^{2+}$ ions (blue spheres 1, 2 and 3), dimethylallyl-Sthiolodiphosphate (DMSPP, yellow) and isopentenyl diphosphate (IPP, green). Metal coordination and hydrogen bond interactions are indicated by solid blue and dotted magenta lines, respectively. The diphosphate group of DMSPP is oriented for abstraction of the pro-R hydrogen from IPP following the chain elongation reaction. Reprinted with permission from Hosfield et al. ${ }^{32}$ Copyright 2004 The American Society for Biochemistry \& Molecular Biology. sites, the relative acidities of their conjugate acid forms are comparable to those of amino acid side chains that serve as more traditional general acids or general bases. For example, the carboxylic acid side chains of glutamic acid and aspartic acid have $\mathrm{pKa}$ values of $\sim 4$, and the imidazolium side chain of histidine has a $\mathrm{pKa}$ value of $\sim 6$ in aqueous solution. In comparison, the second and third ionizations of pyrophosphoric acid have $\mathrm{pKa}$ values of 2.0 and 6.6, and the first and second ionizations of phosphoric acid have $\mathrm{pKa}$ values of 2.1 and 7.2 (Figure 2). ${ }^{35}$

Occasionally participating in enzyme acid-base chemistry are the side chains of cysteine $(\mathrm{pKa} \sim 8.3)$, lysine $(\mathrm{pKa} \sim 10.5)$, tyrosine $(\mathrm{pKa} \sim 10.9)$ and arginine $(\mathrm{pKa} \sim 12.5)$, and the $\mathrm{pKa}$ values for these basic side chains are comparable to those of the third ionization of phosphoric acid (12.7) or the fourth ionization of pyrophosphoric acid (9.4). As with the $\mathrm{pKa}$ values of amino acid side chains, the $\mathrm{pKa}$ values of $\mathrm{P}_{\mathrm{i}}$ and $\mathrm{PP}_{\mathrm{i}}$ can be modulated by their environment and optimized for catalytic function. From the perspective of this chemistry, then, it is quite reasonable to consider the possibility of $\mathrm{PP}_{\mathrm{i}}$ in general basegeneral acid catalysis in a terpenoid cyclase active site. Indeed, there is ample precedent for comparable functions of phosphate derivatives in biological and nonbiological catalysis. Selected examples are summarized below.

\section{Kynureninase}

Also known as L-kynurenine hydrolase, this enzyme utilizes pyridoxal$5^{\prime}$-phosphate (PLP) as a cofactor to catalyze a retro-Claisen reaction yielding products alanine and anthranilic acid (Figure 4$){ }^{36}$ The first step of catalysis is a trans-aldimination with the internal aldimine to generate an external aldimine. The side chain of K227 serves as a general base to deprotonate $\mathrm{C} \alpha$ and then reprotonate $\mathrm{C} 4{ }^{\prime}$ to yield a kynurenine ketamine. ${ }^{37}$ Subsequent addition of a water molecule to the $\gamma$-carbonyl group of ketamine yields a gem-diol; cleavage of the

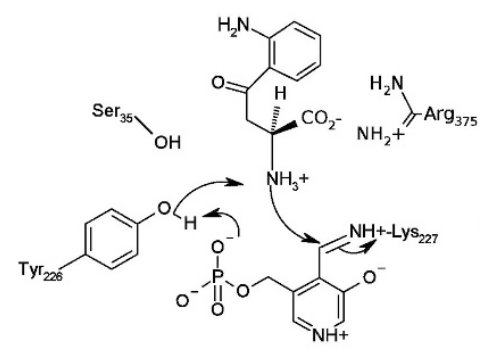

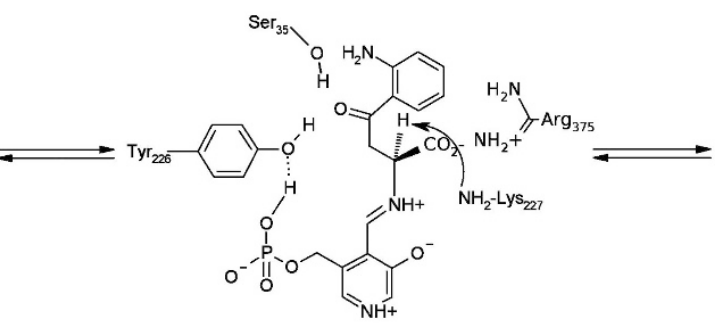

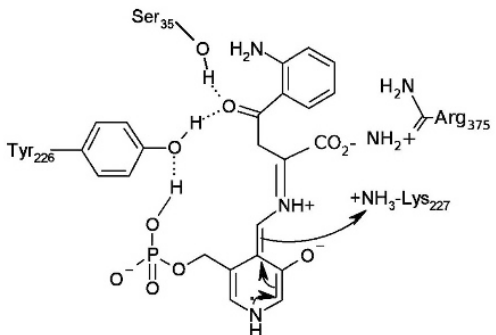

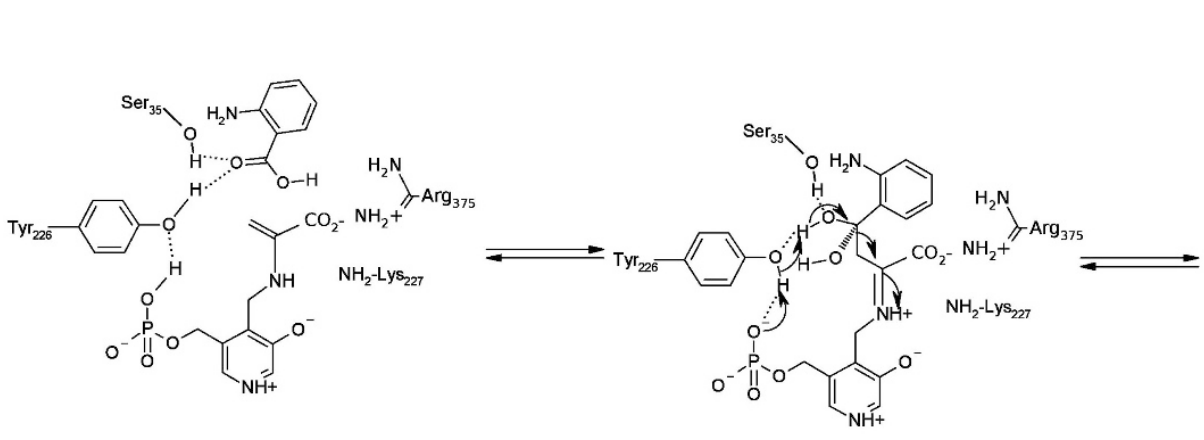

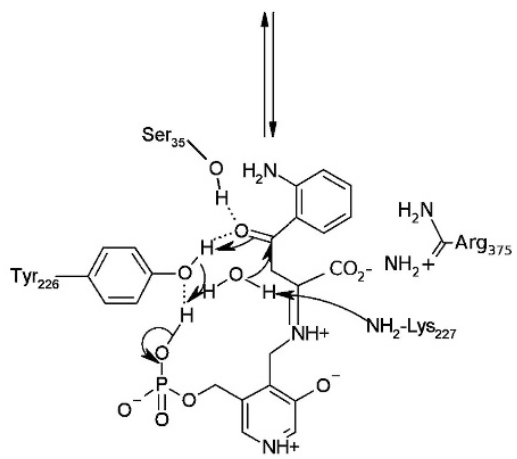

Figure 4 Proposed catalytic mechanism of kynureninase. The pyridoxal-5'-phosphate (PLP)-phosphate serves as a general base-general acid in the activation of Y226 for catalysis. Hydrolysis of the enamine product shown ultimately yields product alanine. Reprinted with permission from Phillips. ${ }^{46}$ Copyright 2015 Elsevier BV. 


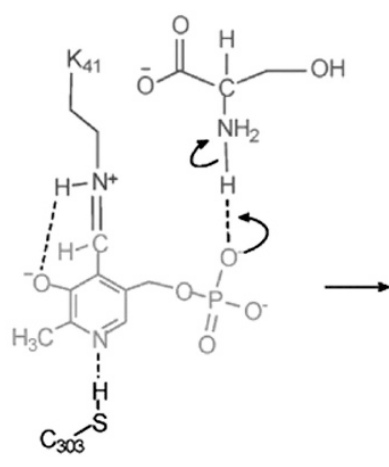

(1)

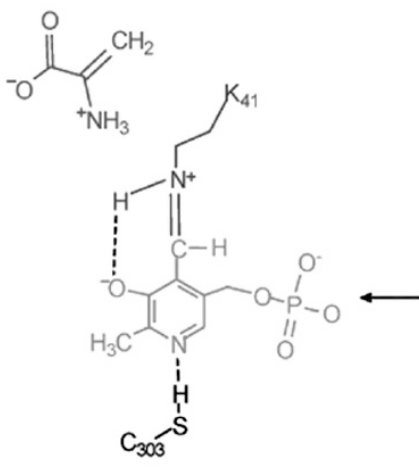

(6)

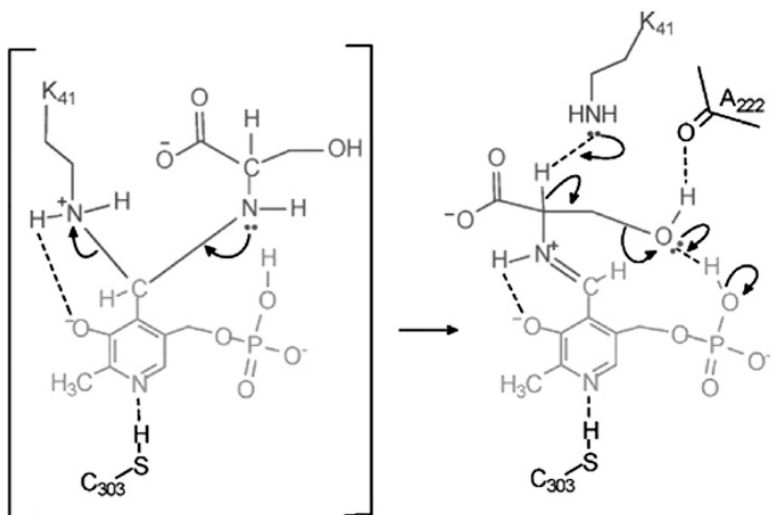

(2)

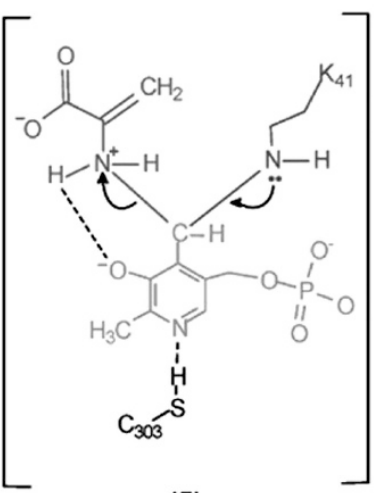

(5)
(3)

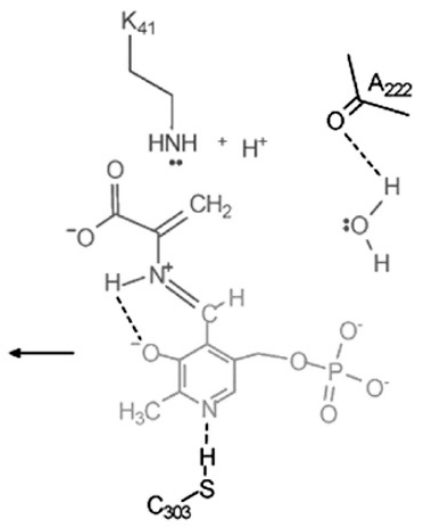

(4)

Figure 5 Proposed catalytic mechanism of serine dehydratase. The serine substrate is red, the pyridoxal-5'-phosphate (PLP) cofactor is green, and the catalytic lysine is blue. The PLP phosphate group serves as a general base-general acid during the course of the reaction. Product aminoacrylate (frame 6 , red) is ultimately hydrolyzed nonenzymatically to form pyruvate and ammonia. Reprinted with permission from Yamada et al. ${ }^{50}$ Copyright 2015 American Chemical Society. A full color version of this figure is available at The Journal of Antibiotics journal online.

$\mathrm{C} \beta-\mathrm{C} \gamma$ bond results in a carboxylic acid product and an enamine intermediate. ${ }^{38}$ Protonation of this enamine at $\mathrm{C} \beta$ yields pyruvate ketamine, which leads to an alanine quinonoid after deprotonation at $\mathrm{C}^{\prime}$. A final protonation at the quinonoid $\mathrm{C} \alpha$ gives the final products L-alanine and aldimine. ${ }^{39-41}$

Crystal structures of human kynureninase ${ }^{41,42}$ reveal that N333, Y275 and S332 are located in the active site and serve as potential hydrogen bond partners for the ligand. The hydrophobic side chains of I110, W305, F306 and F314 form a pocket that accommodates the substrate aromatic group. The $\delta$-oxygen of N333 and the backbone amide of S332 both hydrogen bond with the phosphate group of PLP. The imidazole ring of $\mathrm{H} 102$ provides a $\pi$-stacking partner for the ligand. The structure of the human enzyme complexed with 3-hydroxykynurenine shows that N333 also hydrogen bonds with the C3 hydroxyl group of the inhibitor. Accordingly, the side chains of N333, S332 and H102 dictate specificity for ligand binding.

The position and orientation of a strictly conserved tyrosine, Y275 (or Y226 in the homologous enzyme from Pseudomonas fluorescens ${ }^{43}$ ), led to the suggestion that the phosphate group of PLP serves as a general base in catalysis. Given that the side chain of Y226 hydrogen bonds to the phosphate group of PLP, it is hypothesized that the phosphate group of PLP abstracts a proton from Y226 in the first step of the reaction, and donates this proton back to Y226 in a subsequent step (Figure 4).

The Y226F mutant was prepared to test this hypothesis. ${ }^{44}$ The mutation resulted in a reduction of the $k_{\text {cat }}$ by about 2800 -fold for the substrate L-kynurenine, from $16 \mathrm{~s}^{-1}$ for the wild-type enzyme to $5.8 \times 10^{-3} \mathrm{~s}^{-1}$ for Y226F kynureninase. The $k_{\mathrm{cat}} / K_{\mathrm{m}}$ saw a 375 -fold reduction from $2.0 \times 10^{5} \mathrm{M}^{-1} \mathrm{~s}^{-1}$ for the wild-type enzyme to $530 \mathrm{M}^{-1} \mathrm{~s}^{-1}$ for Y226F kynureninase. To study complexation of the wild-type enzyme with 5-bromodihydrokynurenine, ${ }^{31} \mathrm{P}$-nuclear magnetic resonance (NMR) spectroscopy was used to demonstrate upfield spectroscopic shifts from 4.5 p.p.m. to 5.0, 3.3 and 2.0 p.p.m. on complex formation. These shifts indicated that the phosphate group of PLP undergoes a change in ionization state from dianionic to monoanionic. However, the Y226F mutant does not exhibit these ${ }^{31} \mathrm{P}$ NMR spectroscopic shifts; coupled with the dramatic reduction in turnover for the mutant enzyme, these results suggest that without the Y226 hydrogen bond the PLP phosphate group cannot acquire a proton. ${ }^{45,46}$ The residual activity was hypothesized to be due to a water-mediated hydride shift in the absence of Y226. ${ }^{44}$ These data support the proposed role of the PLP phosphate group as the general base that deprotonates Y226 in catalysis. 


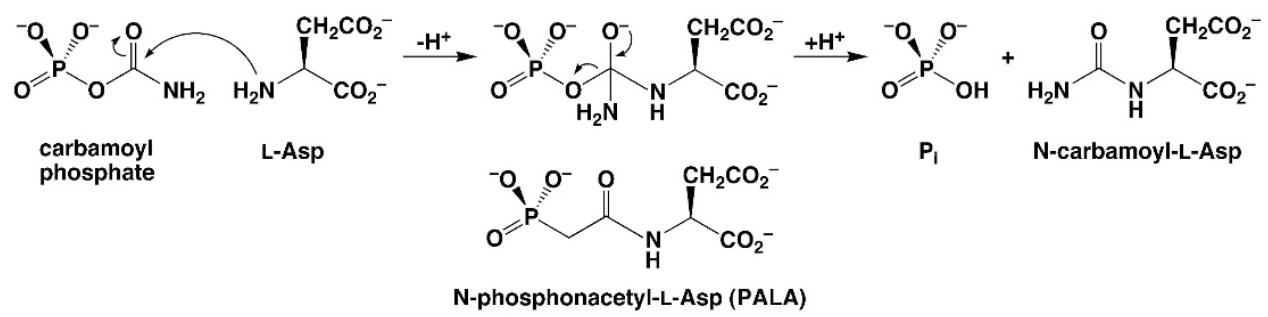

Figure 6 Aspartate transcarbamoylase (ATCase) catalyzes the condensation of carbamoyl phosphate with L-aspartate to yield $\mathrm{N}$-carbamoyl-L-aspartate plus inorganic phosphate $\left(P_{\mathrm{i}}\right)$. The bisubstrate analog $N$-phosphonacetyl-L-aspartate (PALA) mimics structural features of both substrates and is a potent ATCase inhibitor. The binding of PALA reveals key intermolecular and intramolecular interactions that suggest a catalytic function for the phosphate group (Figure 7).



Figure 7 The tetrahedral intermediate in the reaction catalyzed by aspartate transcarbamoylase adopts a 6 -membered ring chair-like conformation. ${ }^{56}$ This conformation enables the phosphate leaving group to serve as a general base, and intramolecular proton transfer facilitates collapse of the tetrahedral intermediate in this example of substrate-assisted catalysis. A full color version of this figure is available at The Journal of Antibiotics journal online.

\section{L-Serine dehydratase}

Eukaryotic L-serine dehydratase $(\mathrm{SDH})$ is a PLP-dependent enzyme that catalyzes the dehydration of L-serine to form pyruvate and ammonia as the final products. ${ }^{47} \mathrm{SDH}$ uses the phosphate group of PLP as a general base in the first step of catalysis.

The catalytic mechanism of SDH is illustrated in Figure 5. First, $\mathrm{L}$-serine enters the active site, with its $\alpha$-amino group forming a hydrogen bond with the phosphate group of PLP.48 The phosphate group then abstracts a proton from the $\alpha$-amino group, forming the free base, which then attacks the $\mathrm{C}^{\prime}$ atom to form a gem-diamine intermediate. Following release of the PLP cofactor from K41 to form a PLP-serine aldimine intermediate, the PLP phosphate group serves as a general acid and donates a proton to the serine hydroxyl group, thereby enabling its departure as a water molecule in concert with the K41-mediated abstraction of the $\mathrm{C} \alpha-\mathrm{H}$ proton. The resulting PLP-aminoacrylate intermediate undergoes nucleophilic attack by K41 at $\mathrm{C}^{\prime}$, and the resulting tetrahedral gem-diamine intermediate collapses to yield amino acrylate and enzyme-bound PLP; aminoacrylate then undergoes nonenzymatic hydrolysis to yield pyruvate and ammonia. $^{49}$

Comparison of the crystal structure of SDH from rat liver with structures of other PLP-dependent enzymes suggests five distinct fold families that utilize the general acid-general base function of the PLP phosphate group. ${ }^{50}$ In SDH, the N1 atom of PLP is adjacent to a neutral residue and is not protonated, and the phosphate group sits in a neutral pocket largely defined by a tetraglycine loop. Since the phosphate group does not interact with any charged amino acids, it likely exists predominantly as the monoanion, $\mathrm{ROPO}_{3} \mathrm{H}^{-}$. All related PLP-dependent enzymes in this group cleave the Ser/Thr C $\beta$-O $\gamma$ bond of serine or threonine. These enzymes belong to the Type II family, which generally catalyze elimination or substitution reactions at the $\beta$-carbon. The Type I family is the largest family and contains enzymes that catalyze transaminations, decarboxylations and $\beta$-eliminations. The remaining three families are much smaller and more specific with type III containing alanine racemase, type IV containing D-amino acid aminotransferases and type $\mathrm{V}$ containing glycogen phosphorylase. It is likely that in these families, too, the PLP phosphate group serves as a general base-general acid in catalysis, for example, as established for glycogen phosphorylase. ${ }^{51}$

\section{Aspartate transcarbamoylase}

The condensation of L-aspartate and carbamoyl phosphate to form $N$-carbamoyl-L-aspartate and $\mathrm{P}_{\mathrm{i}}$ is the first committed step in the biosynthesis of pyrimidine nucleotides in E. coli, and this reaction is catalyzed by the allosteric enzyme aspartate transcarbamoylase (ATCase)..$^{52-54}$ Numerous crystal structures of ATCase have been determined in both $\mathrm{T}$ and $\mathrm{R}$ states with various ligands bound. One such ligand, $\mathrm{N}$-phosphonacetyl-L-aspartate (PALA), is a bisubstrate analog in which it mimics structural features of both substrates (Figure 6). Various details of the catalytic mechanism have been studied and reviewed, ${ }^{52-54}$ and critical structure-mechanism relationships derive from the crystal structure of the ATCase-PALA complex. ${ }^{55}$ Gouaux et al. ${ }^{56}$ used this crystal structure as a template to model the binding of the tetrahedral intermediate. Intriguingly, this study suggested that ideal, 6-membered ring chair-like geometry would support intramolecular proton abstraction by the departing phosphate group to facilitate collapse of the tetrahedral intermediate (Figure 7).

\section{CATALYSIS BY PHOSPHATE IN ORGANIC SYNTHESIS}

The participation of phosphate or phosphoric acid in catalysis is not limited to enzymes, but also includes small-molecule systems that function in non-aqueous solvents. Specifically, derivatives of phosphoric acid readily serve as general acid-general base catalysts (that is, Brønsted acid-Brønsted base catalysts) in organic synthesis. For example, a 1,1'-bi-2-naphthyl-derived phosphoric acid such as that illustrated in Figure 8a can function as a general acid to protonate a reactant, and as a general base to deprotonate a reaction intermediate in a subsequent reaction step.

Consider the Pictet-Spengler reaction (Figure 8a). This is an important reaction used in organic synthesis to generate tetrahydro$\beta$-carboline skeletons found in alkaloid natural products. The mechanism of this reaction requires protonation of an iminium intermediate to facilitate an electrophilic aromatic substitution reaction on the indole ring, and then deprotonation of the tertiary carbocation intermediate to yield the $\beta$-carboline product (Figure $8 \mathrm{~b}$ ). Numerous general acids-general bases will catalyze this reaction, such as acetic acid in methylene chloride. ${ }^{57}$ Interestingly, the Pictet-Spengler reaction is also catalyzed by an enzyme in plant alkaloid biosynthesis. The three-dimensional crystal structure of a 'Pictet-Spenglerase', strictosidine synthase, as well as kinetic isotope effects measured using a specifically deuterated substrate, indicate that E309 serves as a general acid-general base in this reaction (Figure 9). ${ }^{58}$ 
a<smiles>NCCc1c[nH]c2ccccc12</smiles>

1<smiles>O=C(O)CC1CCCCC1=O</smiles>

2

b
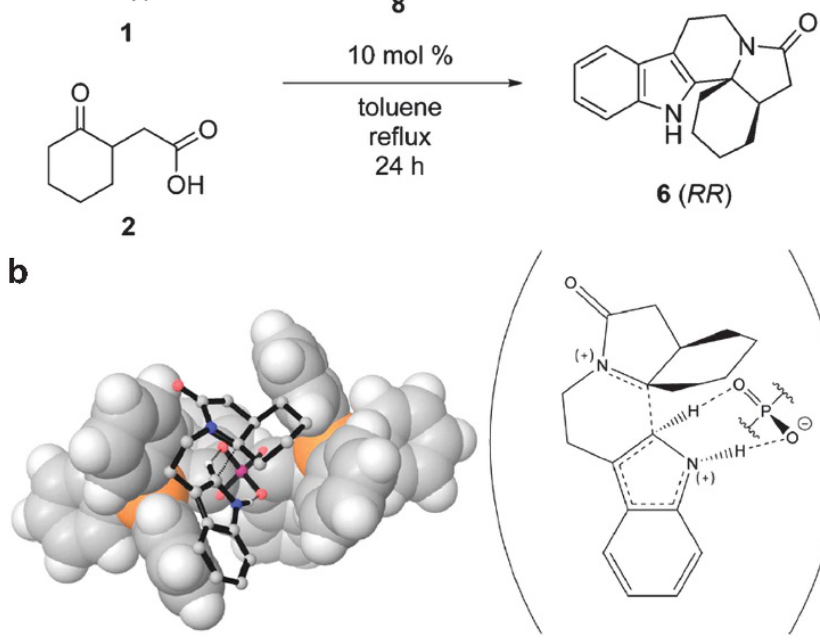

$6(R R)$

Just as acetic acid or E309 can catalyze the Pictet-Spengler reaction in organic solvent or in an enzyme active site, respectively, so too can phosphoric acid derivatives. For example, 1,1'-bi-2-naphthyl derivatives such as 3,3'-bis-(triphenylsilyl)-1,1'-bi-2-naphthol phosphoric acid catalyze this reaction in organic solvent (Figure 8a). ${ }^{59-61}$ The final step of this reaction involves deprotonation of a tertiary carbocation (Figure 8b), which is analogous to the final step of a reaction catalyzed by a terpenoid synthase. If a phosphate derivative can direct the final $\mathrm{C}-\mathrm{H}$ deprotonation in a Pictet-Spengler reaction, then it is reasonable to suggest that the $\mathrm{PP}_{\mathrm{i}}$ anion could direct the final $\mathrm{C}-\mathrm{H}$ deprotonation of a terpenoid cyclization cascade, as proposed for pentalenene synthase in Figure 1.

\section{CONCLUDING REMARKS}

Given the precedent for the function of phosphate and its derivatives in general base-general acid catalysis in enzyme and non-enzyme systems, and given the general dearth of amino acid side chains that could serve general base-general acid functions in terpenoid cyclase active sites, it appears more likely than not that the $\mathrm{PP}_{\mathrm{i}}$ co-product serves this role in terpenoid cyclization cascades. ${ }^{23,25-29}$ An alternative possibility would be a water molecule trapped in the active site; however, since such a water molecule could prematurely quench carbocation intermediates in catalysis, it would have to be located and restrained so as to be chemically inert. Moreover, often there is insufficient residual volume for water binding once the substrate has bound in a terpenoid cyclase active site: enclosed active site volumes are typically just slightly larger than substrate volumes, thereby ensuring a snug fit between the template and the flexible isoprenoid diphosphate substrate. ${ }^{62,63}$

Figure 8 (a) Pictet-Spengler reaction of tryptamine 1 and (2-oxocyclohexyl) acetic acid 2 catalyzed by 1,1'-bi-2-naphthyl (BINOL) derivative 3,3'-bis(triphenylsilyl)-1,1'-bi-2-naphthol phosphoric acid 8. (b) Calculated transition state structure for the Pictet-Spengler reaction of $\mathbf{1}$ and 2 (stick figures) catalyzed by $\mathbf{8}$ (van der Waals surface). The molecular scheme on the right illustrates the proton abstraction by phosphate that quenches the carbocation intermediate. Reprinted with permission from Overvoorde et al. ${ }^{61}$ Copyright 2015 American Chemical Society.

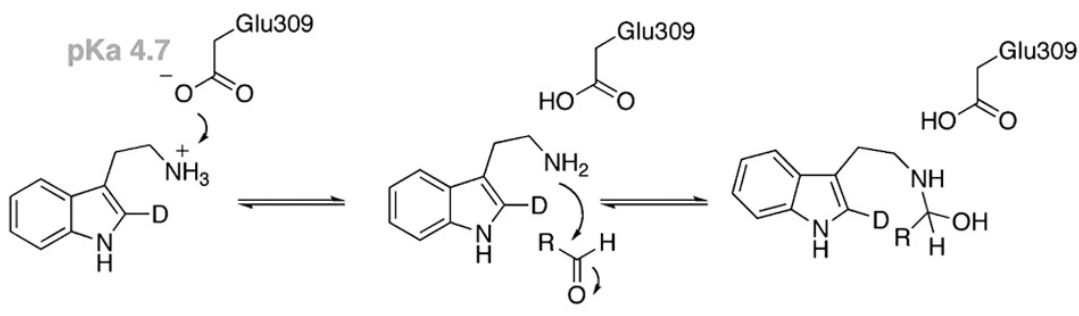<smiles>[R]C1NCCc2c1[nH]c1ccccc21</smiles>

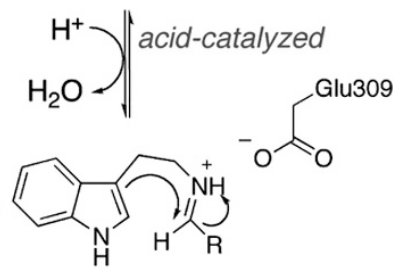

Figure 9 Mechanism of strictosidine synthase, a 'Pictet-Spenglerase', illustrating the general acid-general base function of E309 as determined through kinetic isotope effects using a specifically deuterated substrate. The mechanism of this reaction is identical to that proposed for the general acid-catalyzed reaction in organic synthesis. Reprinted with permission from Maresh et al. ${ }^{58}$. Copyright 2008 American Chemical Society. A full color version of this figure is available at The Journal of Antibiotics journal online. 


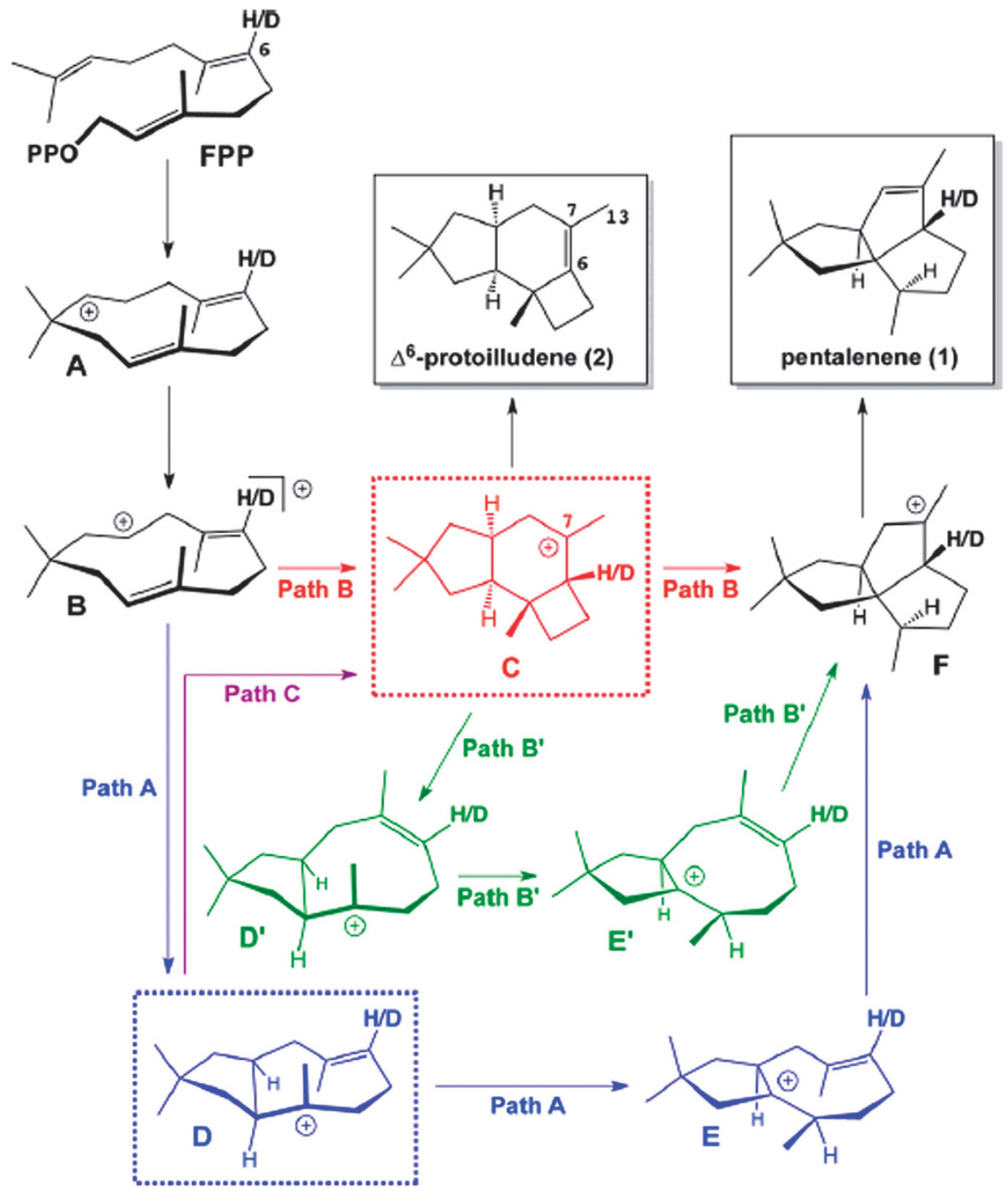

Figure 10 Alternative pentalenene synthase cyclization pathways proceeding through the 7-protoilludyl cation intermediate $\mathbf{C}$ predicted by quantum chemical calculations ${ }^{65}$ and supported by experimentally measured kinetic isotope effects. ${ }^{64}$ Reprinted with permission from Zu et al. ${ }^{64}$. Copyright 2012 American Chemical Society.

Elegant quantum chemical calculations have been used to study the pentalenene synthase mechanism, including the role of $\mathrm{PP}_{\mathrm{i}}$ as the general base that terminates the cyclization cascade. ${ }^{64}$ These calculations correctly predict a kinetic isotope effect measured for the partitioning of products pentalenene and $\Delta^{6}$-protoilludene (Figure 10), so a $\mathrm{PP}_{\mathrm{i}}$ general base can direct the formation of major and minor cyclization products. Notably, these and earlier ${ }^{65}$ quantum chemical calculations point toward alternative cyclization pathways involving the 7-protoilludyl cation as a critical intermediate (structure C in Figure 10), so the details of the pentalenene synthase mechanism remain a topic of current interest. Net 1,4-, 1,5-, and 1,6-proton transfers may occur in some terpenoid cyclase mechanisms without requiring the participation of $\mathrm{PP}_{\mathrm{i}}$ or the enzyme, ${ }^{66}$ so the role of $\mathrm{PP}_{\mathrm{i}}$ in such systems would be less extensive. It is important to note, too, that intramolecular proton or hydride transfer mechanisms can sometimes be ruled out through experiments demonstrating the direct incorporation of a solvent-derived proton in the cyclization product. ${ }^{67} \mathrm{X}$-ray crystal structures reveal that proton transfers of this sort could be mediated by the $\mathrm{PP}_{\mathrm{i}}-\mathrm{Mg}^{2+}{ }_{3}$-complex and $\mathrm{Mg}^{2+}$-bound water molecules. ${ }^{34}$
In closing, given the chemical and functional parallels between the phosphate anions of $\mathrm{P}_{\mathrm{i}}$ or $\mathrm{PP}_{\mathrm{i}}$ and the carboxylate anions of aspartate or glutamate side chains, it is somewhat surprising that there are not more examples of general base-general acid catalysis by phosphatephosphoric acid derivatives in enzyme mechanisms. Such anions, whether generated through the chemistry of catalysis or present simply as buffer components, are not necessarily innocent bystanders in enzyme structure and chemistry. In terpenoid synthase structure and mechanism, the metal-coordinated $\mathrm{PP}_{\mathrm{i}}$ co-product is the most likely source of Brønsted base-acid functionality in the hydrophobic enzyme active site. Future experimental and computational studies will undoubtedly illuminate further details of this function and its contribution to catalysis.

\section{CONFLICT OF INTEREST}

The authors declare no conflict of interest.

\section{ACKNOWLEDGEMENTS}

We thank the National Institutes of Health for grant GM56838 in support of this research. DWC thanks the Radcliffe Institute for Advanced Study for the Elizabeth S and Richard M Cashin Fellowship. 
1 Cane, D. E. Isoprenoid biosynthesis. Stereochemistry of the cyclization of allylic pyrophosphates. Acc. Chem. Res. 18, 220-226 (1985).

2 Croteau, R. \& Cane, D. E. Monoterpene and sesquiterpene cyclases. Methods Enzymol. 110, 383-405 (1985).

3 Cane, D. E. Enzymatic formation of sesquiterpenes. Chem. Rev. 90, 1089-1103 (1990).

4 Allemann, R. K. Chemical wizardry? The generation of diversity in terpenoid biosynthesis. Pure Appl. Chem. 80, 1791-1798 (2008)

5 Austin, M. B., O'Maille, P. E. \& Noel, J. P. Evolving biosynthetic tangos negotiate mechanistic landscapes. Nat. Chem. Biol. 4, 217-222 (2008)

6 Wendt, K. U. \& Schulz, G. E. Isoprenoid biosynthesis: manifold chemistry catalyzed by similar enzymes. Structure 6, 127-133 (1998).

7 Lesburg, C. A., Caruthers, J. M., Paschall, C. M. \& Christianson, D. W. Managing and manipulating carbocations in biology: terpenoid cyclase structure and mechanism. Curr. Opin. Struct. Biol. 8, 695-703 (1998).

8 Tholl, D. Terpene synthases and the regulation, diversity and biological roles of terpene metabolism. Curr. Opin. Plant Biol. 9, 297-304 (2006).

9 Christianson, D. W. Structural biology and chemistry of the terpenoid cyclases. Chem Rev. 106, 3412-3442 (2006).

10 Christianson, D. W. Unearthing the roots of the terpenome. Curr. Opin. Chem. Biol. 12 141-150 (2008).

11 Cane, D. E., Rossi, T., Tillman, A. M. \& Pachlatko, J. P. Stereochemical studies of isoprenoid biosynthesis. Biosynthesis of pentalenolactone from $\left[\mathrm{U}^{13}{ }^{13} \mathrm{C}_{6}\right]$ glucose and $\left[6-{ }^{2} \mathrm{H}_{2}\right]$ glucose. J. Am. Chem. Soc. 103, 1838-1843 (1981).

12 Cane, D. E. \& Tillman, A. M. Pentalenene biosynthesis and the enzymatic cyclization of farnesyl pyrophosphate. J. Am. Chem. Soc. 105, 122-124 (1983).

13 Cane, D. E. et al. Biosynthesis of pentalenene and pentalenolactone. J. Am. Chem. Soc 112, 4513-4524 (1990).

14 Koe, B. K., Sobin, B. A. \& Celmer, W. D. PA 132, a new antibiotic. I. Isolation and chemical properties. Antibiot. Annu. 672-675 (1957).

15 Hartmann, S., Neeff, J., Heer, U. \& Mecke, D. Arenaemycin (pentalenolactone): a specific inhibitor of glycolysis. FEBS Lett. 93, 339-342 (1978).

16 Mann, K. \& Mecke, D. Inhibition of spinach glyceraldehyde-3-phosphate dehydrogenases by pentalenolactone. Nature 282, 535-536 (1979).

17 Cane, D. E., Abell, C. \& Tillman, A. M. Pentalenene biosynthesis and the enzymatic cyclization of farnesyl pyrophosphate: proof that the cyclization is catalyzed by a single enzyme. Bioorg. Chem. 12, 312-328 (1984).

18 Cane, D. E. et al. Pentalenene synthase. Purification, molecular cloning, sequencing, and high-level expression in Escherichia coli of a terpenoid cyclase from Streptomyces UC5319. Biochemistry 33, 5846-5857 (1994).

19 Lesburg, C. A., Zhai, G., Cane, D. E. \& Christianson, D. W. Crystal structure of pentalenene synthase: mechanistic insights on terpenoid cyclization reactions in biology, Science 277, 1820-1824 (1997).

20 Seemann, M., Zhai, G., Umezawa, K. \& Cane, D. Pentalenene Synthase. Histidine-309 is not required for catalytic activity. J. Am. Chem. Soc. 121, 591-592 (1999).

21 Starks, C. M., Back, K., Chappell, J. \& Noel, J. P. Structural basis for cyclic terpene biosynthesis by tobacco 5-epi-aristolochene synthase. Science 277 1815-1820 (1997).

22 Seemann, M. et al. Pentalenene synthase. Analysis of active site residues by sitedirected mutagenesis. J. Am. Chem. Soc. 124, 7681-7689 (2002).

23 Caruthers, J. M., Kang, I., Rynkiewicz, M. J., Cane, D. E. \& Christianson, D. W. Crystal structure determination of aristolochene synthase from the blue cheese mold, Penicillium roqueforti. J. Biol. Chem. 275, 25533-25539 (2000).

24 Felicetti, B. \& Cane, D. E. Aristolochene synthase: mechanistic analysis of active site residues by site-directed mutagenesis. J. Am. Chem. Soc. 126, 7212-7221 (2004).

25 Shishova, E. Y. Di Costanzo, L. Cane, D. E \& Christianson, D. W. X-ray crysta structure of aristolochene synthase from Aspergillus terreus and evolution of templates for the cyclization of farnesyl diphosphate. Biochemistry 46, 1941-1951 (2007).

26 Miller, D. J. \& Allemann, R. K. Sesquiterpene synthases: passive catalysts or active players? Nat. Prod. Rep. 29, 60-71 (2012).

27 Faraldos, J. A., Gonzalez, V. \& Allemann, R. K. The role of aristolochene synthase in diphosphate activation. Chem. Commun. 48, 3230-3232 (2012).

28 Köksal, M., Zimmer, I., Schnitzler, J.-P. \& Christianson, D. W. Structure of isoprene synthase illuminates the chemical mechanism of teragram atmospheric carbon emission. J. Mol. Biol. 402, 363-373 (2010)

29 Faraldos, J. A. et al. Probing the mechanism of 1,4-conjugate elimination reactions catalyzed by terpene synthases. J. Am. Chem. Soc. 134, 20844-20848 (2012).

30 Aaron, J. A. \& Christianson, D. W. Trinuclear metal clusters in catalysis by terpenoid synthases. Pure Appl. Chem. 82, 1585-1597 (2010).

31 Rynkiewicz, M. J., Cane, D. E. \& Christianson, D. W. Structure of trichodiene synthase from Fusarium sporotrichiodes provides mechanistic inferences on the terpene cyclization cascade. Proc. Natl Acad. Sci. USA 98, 13543-13548 (2001).

32 Hosfield, D. J. et al. Structural basis for bisphosphonate-mediated inhibition of isoprenoid biosynthesis. J. Biol. Chem. 279, 8526-8529 (2004).

33 Tarshis, L. C., Yan, M., Poulter, C. D. \& Sacchettini, J. C. Crystal structure of recombinant farnesyl diphosphate synthase at $2.6-\AA$ r resolution. Biochemistry 33 10871-10877 (1994).

34 Chen, M. et al. Mechanistic insights from the binding of substrate and carbocation intermediate analogues to aristolochene synthase. Biochemistry 52, 5441-5453 (2013).

35 Tymoczko, J. L., Berg, J. M. \& Stryer, L. Biochemistry: A Short Course, 2nd edn p. A3 (W. H. Freeman and Company, New York, USA, 2013).

36 Phillips, R. S., Sundararaju, B. \& Koushik, S. V. The catalytic mechanism of kynureninase from Pseudomonas fluorescens: evidence for transient quinonoid and ketimine intermediates from rapid-scanning stopped-flow spectrophotometry Biochemistry 37, 8783-8789 (1998).

37 Phillips, R. S. \& Dua, R. Stereochemistry and mechanism of aldol reactions catalyzed by kynureninase. J. Am. Chem. Soc. 113, 7385-7388 (1991).

38 Koushik, S. V., Moore, J. A. III, Sundararaju, B. \& Phillips, R. S. The catalytic mechanism of kynureninase from Pseudomonas fluorescens: insights from the effects of $\mathrm{pH}$ and isotopic substitution on steady-state and pre-steady-state kinetics. Biochemistry 37, 1376-1382 (1998).

39 Gawandi, V. B., Liskey, D., Lima, S. \& Phillips, R. S. Reaction of Pseudomonas fluorescens kynureninase with $\beta$-benzoyl-L-alanine: detection of a new reaction intermediate and a change in rate-determining step. Biochemistry 43, 3230-3237 (2004).

40 Kumar, S., Gawandi, V. B., Capito, N. \& Phillips, R. S. Substituent effects on the reaction of $\beta$-benzoylalanines with Pseudomonas fluorescens kynureninase. Biochemistry 49, 7913-7919 (2010).

41 Lima, S., Kumar, S., Gawanadi, B., Momany, C. \& Phillips, R. S. Crystal structure of the Homo sapiens kynurenine-3-hydroxyhippuric acid inhibitor complex: Insights into the molecular basis of kynurenine substrate specificity. J. Med. Chem. 52 , 389-396 (2009).

42 Lima, S., Khristoforov, R., Momany, C. \& Phillips, R. S. Crystal structure of Homo sapiens kynureninase. Biochemistry 46, 2735-2744 (2007).

43 Momany, C., Levdikov, V., Blagova, L., Lima, S. \& Phillips, R. S. Three-dimensional structure of kynureninase from Pseudomonas fluorescens. Biochemistry 43 $1193-1203$ (2004)

44 Phillips, R. S., Scott, I., Paulose, R., Patel, A. \& Barron, T. C. The phosphate of pyridoxal-5'-phosphate is an acid/base catalyst in the mechanism of Pseudomonas fluorescens kynureninase. FEBS J. 281, 1100-1109 (2014).

45 Phillips, R. S. Structure and mechanism of kynureninase. Arch. Biochem. Biophys. 544, 69-74 (2014)

46 Phillips, R. S. Chemistry and diversity of pyridoxal-5'-phosphate dependent enzymes. Biochim. Biophys. Acta 1854, 1167-1174 (2015).

47 Flint, D. H. \& Allen, R. M. Iron-sulfur proteins with nonredox functions. Chem. Rev. 96 2315-2334 (1996)

48 Hwang, B. Y., Cho, B. K., Yun, K., Koteshwar, K. \& Kim, B. G. Revisit of aminotransferases in the genomic era and its application to biocatalysis. J. Mol. Catal. B Enzym. 37, 47-55 (2005).

49 Urusova, D. V. et al. Crystal Structure of D-serine dehydratase from Escherichia coli. Biochim. Biophys. Acta. 1824, 422-432 (2012).

50 Yamada, T. et al. Crystal structure of serine dehydratase from rat liver. Biochemistry 42 12854-12865 (2003).

51 Johnson, L. N., Hu, S.-H. \& Barford, D. Catalytic mechanism of glycogen phosphorylase. Faraday Discuss 93, 131-142 (1992).

52 Kantrowitz, E. R. Allostery and cooperativity in Escherichia coli aspartate transcarbamoylase. Arch. Biochem. Biophys. 519, 81-90 (2012).

53 Lipscomb, W. N. \& Kantrowitz, E. R. Structure and mechanisms of Escherichia coli aspartate transcarbamoylase. Acc. Chem. Res. 45, 444-453 (2012).

54 Lipscomb, W. N. Aspartate transcarbamylase from Escherichia coli: activity and regulation. Adv. Enzymol. Relat. Areas Mol. Biol. 68, 67-151 (1994).

55 Krause, K. L., Volz, K. W. \& Lipscomb, W. N. $2.5 \AA$ structure of aspartate carbamoyltransferase complexed with the bisubstrate analog $N$-(phosphonacetyl)- $L$ aspartate. J. Mol. Biol. 193, 527-553 (1987).

56 Gouaux, J. E., Krause, K. L. \& Lipscomb, W. N. The catalytic mechanism of Escherichia coli aspartate carbamoyltransferase: a molecular modelling study. Biochem. Biophys. Res. Commun. 142, 893-897 (1987).

57 Zhou, H., Liao, X \& Cook, J. M. Regiospecific, enantiospecific total synthesis of the 12-alkoxy-substituted indole alkaloids, (+)-12-methoxy- $N_{\mathrm{a}}$-methylvellosimine, (+)-12-methoxyaffinisine, and (-)-fuchsiaefoline. Org. Lett. 6, 249-252 (2004).

58 Maresh, J. J. et al. Strictosidine synthase: mechanism of a Pictet-Spengler catalyzing enzyme. J. Am. Chem. Soc. 130, 710-723 (2008).

59 Seayad, J., Seayad, A. M. \& List, B. Catalytic asymmetric Pictet-Spengler reaction. J. Am. Chem. Soc. 128, 1086-1087 (2006).

60 Holloway, C. A., Muratore, M. E., Storer, R. I. \& Dixon, D. J. Direct enantioselective Brønsted acid catalyzed $\mathrm{N}$-acyliminium cyclization cascades of tryptamines and ketoacids. Org. Lett. 12, 4720-4723 (2010).

61 Overvoorde, L. M., Grayson, M. N., Luo, Y. \& Goodman, J. M. Mechanistic insights into a BINOL-derived phosphoric acid-catalyzed asymmetric Pictet-Spengler reaction. J. Org. Chem. 80, 2634-2640 (2015).

62 Rynkiewicz, M. J., Cane, D. E. \& Christianson, D. W. X-ray crystal structures of D100E trichodiene synthase and its pyrophosphate complex reveal the basis for terpene product diversity. Biochemistry 41, 1732-1741 (2002).

63 Köksal, M., Jin, Y., Coates, R. M., Croteau, R. \& Christianson, D. W. Taxadiene synthase structure and evolution of modular architecture in terpene biosynthesis. Nature 469 , 116-120 (2011).

$64 \mathrm{Zu}, \mathrm{L}$. et al. Effect of isotopically sensitive branching on product distribution for pentalenene synthase: support for a mechanism predicted by quantum chemistry. J. Am. Chem. Soc. 134, 11369-11371 (2012).

65 Gutta, P. \& Tantillo, D. J. Theoretical studies on farnesyl cation cyclization: pathways to pentalenene. J. Am. Chem. Soc. 128, 6172-6179 (2006).

66 Hong, Y. J. \& Tantillo, D. J. Feasibility of intramolecular proton transfers in terpene biosynthesis-guiding principles. J. Am. Chem. Soc. 137, 4134-4140 (2015).

67 Miller, D. J. et al. Stereochemistry of eudesmane cation formation during catalysis by aristolochene synthase from Penicillium roqueforti. Org. Biomol. Chem. 6, 2346-2354 (2008). 